\title{
Infection control protocol inside computed tomography suites during COVID-19 outbreak
}

\author{
Beuy Joob ${ }^{1} \cdot$ Viroj Wiwanitkit ${ }^{2,3}$
}

Received: 19 March 2020 / Accepted: 24 March 2020 / Published online: 31 March 2020

(c) Japan Radiological Society 2020

\section{Dear Editor,}

We would like to share ideas on "COVID-19 pneumonia: infection control protocol inside computed tomography suites". Nakajima et al. noted that "CT imaging is helpful for the evaluation of the novel coronavirus disease 2019 (COVID-19) pneumonia. Infection control inside the CT suites is also important to prevent hospital-related transmission of COVID-19 [1]". The role of CT scan in diagnosis of COVID-19 is widely discussed. In our country, Thailand, the second country that COVID-19 appeared [2], the diagnosis of all 272 patients (19 March 2020) is based on PCR diagnosis. The CT finding is negative in many cases of early infection. The infection control in CT room is an important issue, but usually under-recognized. There is usually no sufficient fund for rearrangement of the CT room to correspond to COVID-19. The risk of the radiologists and radiotechnologists who practice in the $\mathrm{CT}$ room is also unknown.

\section{Compliance with ethical standards}

Conflict of interest The authors declare that they have no conflict of interest statement.

\section{References}

1. Nakajima K, Kato H, Yamashiro T, Izumi T, Takeuchi I, Nakajima H, Utsunomiya D. COVID-19 pneumonia: infection control protocol inside computed tomography suites. Jpn J Radiol. 2020. https ://doi.org/10.1007/s11604-020-00948-y(Epub ahead of print).

2. Yasri S, Wiwanitkit V. Editorial: Wuhan coronavirus outbreak and imported case. Adv Trop Med Pub Health Int. 2019;9:1-2.

Publisher's Note Springer Nature remains neutral with regard to jurisdictional claims in published maps and institutional affiliations.
Beuy Joob

beuyjoob@hotmail.com

Sanitation 1 Medical Academic Center, Bangkok, Thailand

2 Dr DY Patil University, Pune, India

3 Hainan Medical University, Haikou, China 